Sitzungsberichte des Plenums

und der problemgebundenen Klassen

der Deutschen Akademie

der Wissenschaften zu Berlin

\title{
Harald Dutz
}

\section{Probleme der}

\section{Organtransplantation}


